\title{
Complex Hydrides - When Powder Diffraction needs Help
}

\author{
Pascal Schouwink and Radovan Černý*
}

\begin{abstract}
Real life' energy-related materials such as solid-state hydrogen storage compounds or components of electrochemical cells are usually polycrystalline, poorly crystallized, highly reactive and dynamic systems. Powder diffraction at modern high brilliance X-ray sources is the most useful tool to investigate such systems because it is easy, fast and extremely versatile with respect to measurement conditions as well as in situ setups. However, it is in the nature of these systems that they undergo processes that cannot be investigated by diffraction alone. The central role in hydrogen storage materials is played by hydrogen itself, the worst X-ray scatterer in the periodic system. Gas release, the purpose of a hydrogen storage material, is not detected by diffraction. Amorphous components are badly characterized. We want to show how a complementary approach combining different methods allows to overcome limitations imposed on powder diffraction by the sample nature of 'real' hydrogen storage materials.
\end{abstract}

Keywords: Complex hydride $\cdot$ Hydrogen storage $\cdot$ Powder diffraction $\cdot$ in situ Studies

Interstitial metal hydrides based on intermetallic alloys have since long been the number one contenders for solid-state hydrogen storage and both X-ray as well as neutron powder diffraction have been indispensable tools for their characterization. Many different intermetallics are nowadays being used as reversible hydrogen storage systems to transport energy. However, their high weight per unit volume imposes severe restrictions when it comes to mobile applications. Thus, intermetallic celebrities such as $\mathrm{LaNi}_{5}{ }^{[1]}$ are used mainly in stationary hydrogen storage tanks; more exotic applications include marine warfare. ${ }^{[2]}$ The most seminal use of solid-state hydrogen storage is in automobiles, ${ }^{[3,4]}$ an application that is far out of reach for any intermetallic interstitial hydride. Complex

*Correspondence: Prof. R Černý

University of Geneva

Laboratory of Crystallography, DPMC

24, Quai Ernest-Ansermet

$\mathrm{CH}-1211$, Geneva 4

Tel.: +41223796450

E-mail: radovan.cerny@unige.ch hydrides in which hydrogen forms covalent bonds, do not suffer from this drawback. The field of complex hydrides is still young, but the number of scientific reports has been skyrocketing during the last 5-10 years. A comprehensive review is given by Walker. ${ }^{[1]}$ Research on light (and not so light) complex hydrides has to overcome a number of difficulties, especially those concerning their accurate characterization. Here we shall focus on the tetrahydroborates; they are based on the complex anion $\mathrm{BH}_{4}^{-}$and are commonly called borohydrides. Developing an application requires comprehensive knowledge, both experimental and theoretical, of all relevant materials. The experimentalists need to characterize their samples as precisely as possible and provide the theoreticians with accurate descriptions for the latter to model, optimize and predict processes and systems. In the field of gas uptake and release, complex hydrides were traditionally characterized by thermodynamic measurements and sorption-desorption experiments. However these experiments provide no information on phase compositions, let alone structural details of the compounds involved. Nowadays state-ofthe-art experiments in the field strongly rely on powder diffraction and in situ studies in versatile environments. The number of reported new structures has skyrocketed since the emergence of modern powder diffraction methods and the user-friendly implementation of direct space methods, which use global optimization algorithms to solve crystal structures; typically structure solution in reciprocal space is next to impossible given the imperfect nature of the samples. Even though the power of powder X-ray diffraction (PXD) at high brilliance sources is overwhelming, the materials and systems contending for hydrogen storage applications impose limits on the applicability of the method. Often systems are out of thermodynamic equilibrium and contain a multitude of amorphous and/or metastable phases which may form already during synthesis. Reactions taking place during the generally used mechanochemical synthesis and during dehydrogenation often lead to poorly crystallized multiphase samples, commonly containing more than one novel unknown phase. The synthetic approaches have been reviewed recently and will not be addressed here. ${ }^{[5,6]}$ Crystallographic headaches caused by poor and complex data are greatly alleviated when complementary experimental information can be provided, e.g. from TGAMS or DTA, from vibrational and NMR spectroscopy, from quasi-elastic neutron scattering (QENS), which provides important structural details, or from theoretical methods which often allow chemically doubtful structural features to be corrected. A complementary and iterative implementation of all experimental methods leads to the detailed experimental description of 'real life' data, that is essential for doing good theoretical work on kinetics, thermodynamics and prediction of new 'real life' candidate storage systems.

\section{In situ Synchrotron Radiation Powder Diffraction}

The most obvious way to study samples that are prepared as fine-crystalline powders is powder diffraction. Only very 
few single crystal studies have been report$\mathrm{ed}^{[7-10]}$ amongst which there is not a single example of a bimetallic compound. Since $\mathrm{X}$-ray scattering requires much shorter acquisition times it is largely preferred to neutron diffraction, which, nevertheless, is obviously the tool of choice when it comes to the localization of hydrogen (deuterium) atoms. Applications in hydrogen storage involve systems that are highly reactive and dynamic; hence, a good time resolution is indispensable. We will show throughout this contribution how X-ray diffraction is complemented by methods other than neutron diffraction to circumvent the lacking information provided on hydrogen. Modern area detectors or curved silicon strip detectors are nowadays available at most synchrotron powder diffraction beamlines, allowing for simple and flexible setups for different kinds of in situ studies.

Two-dimensional detectors provide excellent counting statistics and enable rapid data collection. This allows for a highly accurate determination of diffracted intensities (required for refinement) and a low sensitivity to preferred orientation or sample inhomogeneity (graininess, spatial phase separation), as well as an excellent time resolution. Curved solid-state detectors in turn offer excellent reciprocal space resolution, which is often required to index unknown phases. The most basic in situ setup adapted to scan new potential hydrogen storage systems is a rotating glass capillary under a heat source, such as a gas blower or a cryostream. The simplicity of this setup allows complementary solidstate characterization methods (Raman, EXAFS), and those that investigate gas release (IR, TGA-MS), to be easily coupled to the diffraction experiment. Temperatureresolved studies are essential to study fast reactions involving metastable compounds and intermediate decomposition products that may appear over temperature intervals of a few $\mathrm{K}$ only but are relevant to describe the decomposition process and thus evaluate the feasibility as a storage material. Rehydrogenation can be studied in a more elaborate setup involving a high-pressure gas cell (up to 300 bar $\left.\mathrm{P}\left(\mathrm{H}_{2}\right)\right)^{[11]}$ and measuring the sample in single-crystal sapphire capillaries. Single crystal diffraction spots are easily seen on an area detector and masked before integration. High-energy beamlines make it possible to measure to higher gas pressures (1000 bar) inside steel containers, simulating a storage tank. In situ ball-milling setups are currently being developed to study reactions taking place during mechano-chemical synthesis, ${ }^{[12]}$ as it remains a black box and the resulting powders often are made up of decomposition products.

A note should be made on diffraction studies at non-ambient pressure conditions in diamond anvil cells, where care should be taken to carefully define and model preferred orientation during global optimization. ${ }^{[13]}$

\section{The Limits}

Structures need to be fully characterized to assess a whole system of reactions and changing compositions, which is going to be the working case scenario of any potential hydrogen storage material. Only in the rarest cases is a sample a well crystallized and single phase. ${ }^{[14]}$ The different approaches to structure solution from powder samples are covered in another contribution to this issue ${ }^{[15]}$ and will not be elaborated on here. Most commonly the multi-phase nature and poor crystallinity of the investigated powders lead to significant peak broadening and overlap and prevent structural solution in reciprocal space. Just one example has been reported for borohydrides. ${ }^{[14]}$ Thus it is direct space mode-ling that becomes the dominant method for structure solution. It does not require structure amplitudes to be extracted and can deal better with peak overlap, thus allowing work on low resolution data. ${ }^{[16]}$ In particular, the direct space program Fox ${ }^{[17]}$ has become very popular in the hydride community and has led to structure solutions of unit cells containing as many as 55 independent atoms. ${ }^{[18]}$ Some of the, nevertheless, more commonly occurring problems in complex metal hydrides that can lead to wrong conclusions are mentioned in the following.

\section{Unit Cell Content}

Addition or metathesis reactions taking place during ball milling yield a number of phases of different compositions or even polymorphs of the same stoichiometry. Especially if more than one novel phase is present in the resulting product the unit cell contents may easily be estimated incorrectly. If data and sample quality are sufficient the 'dynamical occupancy correction' ${ }^{[17]}$ implemented in Fox is capable of merging atoms and reducing potentially wrong multiplicities. However, misestimating unit cell contents may also bias the choice of unit cell size, metric or even crystal system, due to the constraints imposed by Wyckoff site multiplicities. Sometimes the resulting structure may provide nearly satisfying Rietveld fits to powder diffraction data and converge to well-balanced interatomic forces in $a b$ initio calculations, but still not correspond to the measured crystal structure and hence not represent the stable polymorph at the given thermodynamic conditions. ${ }^{[19,20]}$ These cases pose severe problems, especially on poor data. To eliminate these error sources it is crucial to prepare various mixtures of different ratios of starting materials. By analyzing the varying phase compositions (relative signal intensities) it is possible to deduce correct unit cell contents. It should be noted that amorphous phases can be present which can simulate wrong phase compositions, meaning that excess fractions of reactants may be in an amorphous state after ball-milling, without having reacted.

\section{Indexing and Space Group}

Determining the correct space group from poorly crystallized multiphase powder samples is extremely challenging. It is virtually impossible without temperatureresolved in situ studies, which allow the separation of different phases by 'decomposition-aided indexing'.[21] While lattice centering is usually detected relatively easily, the choice of space group symmetry elements becomes more challenging, especially due to the fact that the major component determining the space group symmetry is often the orientation of the $\mathrm{BH}_{4}^{-}$anion and hence, the weakest scatterer, hydrogen, itself. Experience is the best tool here. Borohydrides (as molecules), for instance, tend to 'like' screw axes and glide planes due to the different ordering schemes of the tetrahydroborate that are compatible with periodicity. The choice upon inversion symmetry remains the most complicated one, especially when it comes down to assigning disorder in $\mathrm{BH}_{4}$ group, or ordering it on a site of lower symmetry. If the compound undergoes a phase transition, group-subgroup relations can help to decide on centrosymmetry. Order-disorder transitions can lead to large supercells in the ordered phase that can be virtually invisible to X-ray diffraction. However, the transition enthalpy can be quantified by means of DSC, and its magnitude provides information on the character of the transformation. Displacive and order-disorder transitions may be separated this way, as was recently applied in a series of borohydrides crystallizing in the perovskite-type, that show a sequence of displacive as well as order-disorder transitions (Schouwink, pers. comm.). The transition enthalpy of an order-disorder transition assuming complete configurational disorder of the $\mathrm{BH}_{4}$ group yields an entropic contribution of around $T \Delta S=k \ln 2$, as is the case for the $L T-H T$ transition of $\mathrm{NaBH}_{4}$ for instance. More elaborate ways of detecting disorder include solid-state NMR and QENS, which directly probe the dynamics of rotational groups and are capable of discriminating between polar and non-polar disorder by fitting different models containing different jump-motions. In the recent past we have been applying DFT more and more 
to correct space groups, ${ }^{[22,23]}$ when diffraction data and crystal chemistry were lacking information. More specifically, DFT can be resorted to so as to decide amongst a number of supergroups when the phase is solved in a subgroup. The reverse is likewise possible, when structures are solved by exploiting structural analogies to oxides or halides. In these cases, borohydrides commonly crystallize in a subgroup to the topologically related compound, as the non-spherical tetrahydroborate anion introduces additional distortions and ordering schemes. ${ }^{[24]}$ Recent structure solutions on e.g. $\mathrm{KAl}\left(\mathrm{BH}_{4}\right)_{4}{ }^{[25]}$ and $\mathrm{Li}_{3} \mathrm{Cs}_{2}\left(\mathrm{BH}_{4}\right)_{5},{ }^{[24]}$ both in the rare space group $F d d d$, have taught us that large supercells must not be intimidating, if they match higher symmetries.

An important step during the process of structure determination is its validation. Periodic density functional theory calculations are applied here to show that the structural model is in the minimum of its potential energy and that the interatomic forces are balanced. An equally important tool is crystal chemical systematics established by generations of experienced crystallographers. As metal borohydrides are ionocovalent or ionic compounds, the majority of them resemble structures of metal oxides or halides, as mentioned above. Based on this systematic structural similarity, database searches can be performed with the aim of validating the structure of a novel borohydride. Furthermore, the topological analysis of underlying nets in borohydrides provides a means of validating new topologies.

\section{$\mathrm{BH}_{4}$ : Coordination Modes and Orientation - The Role of Vibrational Spectroscopy and DFT}

Structural details such as the bonding configuration of the $\mathrm{BH}_{4}$ to the metal center are information that is largely hidden to X-ray powder diffraction. Two structural characterizations, the cases of $\mathrm{LiBH}_{4}$ and $\mathrm{Mg}\left(\mathrm{BH}_{4}\right)_{2}$, have succeeded in correctly positioning hydrogen atoms without restraints. In both cases however, refinements were performed on single crystal data $^{[7,8]}$ and, in both compounds, the contribution of hydrogen to the scattering of $\mathrm{X}$-rays is important, due to the light-weight counter-cations. Neutron diffraction will more often provide accurate hydrogen positions, but is neither easily accessible nor measurable on metastable samples or intermediate phases that crystallize and/ or decompose/melt rapidly with temperature. Therefore, the metal-center hydrogen bonding scheme is usually retrieved by introducing various antibump-restraints on the rigid body $\mathrm{BH}_{4}$ during Rietveld refinements, having the maximization of

$\mathrm{H}$...H distances as an aim. In solid-state homoleptic borohydrides there exist two main coordination schemes that can mix in different environments. Their idealized configuration is shown in Fig. 1. Internal B-H vibrations are very sensitive to the local environment and can be used to obtain structural information by analyzing the way bands split, especially in the deformation region around $1150-1450 \mathrm{~cm}^{-1}$. The stretching region (ca. $2150-2500 \mathrm{~cm}^{-1}$ ) is subject to significant Fermi-resonances that generally spread over $100 \mathrm{~cm}^{-1}$, which can complicate mode analysis. Vibrational spectroscopy can address two different issues: (i) it can validate and strengthen structural topologies determined by powder diffraction. Genuine frameworks (i.e. containing only bridging hydrogen atoms) and structures built from complex anions of the kind $\left[M_{\mathrm{n}}^{\mathrm{m}+}\left(\mathrm{BH}_{4}\right)_{\mathrm{p}}\right]^{(\mathrm{p}-\mathrm{n} * \mathrm{~m})-}$ (contains both bridging and terminal hydrogen atoms) counterbalanced by, in general, monovalent cations, have specific vibrational signatures in the spectrum. (ii) Different M...H bonding schemes (Fig. 1) have different spectroscopic signatures, thus both IR and Raman spectroscopy can yield valuable information on the local environment of the metal center.[26] Not only can the bonding mode underlay structural topologies, but its geometry can have influence on the dehydrogenation behavior (kinetics and mechanisms) $)^{[27]}$ and is thus essential to characterize accurately in a potential storage material to derive the processes that destabilize the actual $\mathrm{BH}_{4}^{-}$anion and hence dictate the mechanism of $\mathrm{H}_{2}$ formation.

\section{General Topology}

A genuine framework such as tetrahedral $\mathrm{Mg}\left(\mathrm{BH}_{4}\right)_{2}$ or octahedral $\mathrm{Ca}\left(\mathrm{BH}_{4}\right)_{2}$ does not impact significantly on the splitting of B-H stretching vibrations. Complex anions on the other hand are identified by split deformation as well as stretching regions. In the stretching regions the $\mathrm{B}-\mathrm{H}_{t}$ modes pertaining to the terminal hydrogen atoms of the $\mathrm{BH}_{4}$ ligands come to lie at higher frequencies, while the opposite is observed in the deformation region. Here the bending modes of inbound $M-\mathrm{H}-\mathrm{B}$ bridging hydrogen atoms are shifted to higher energies and become very intense due to coupling to $M-\mathrm{H}$ stretching modes. The stoichiometry of a complex anion can further be investigated to validate experimental studies by cluster calculations on isolated anions, e.g. the triangular and tetrahedral variants of $\left[\mathrm{Zn}\left(\mathrm{BH}_{4}\right)_{\mathrm{n}}\right]^{(\mathrm{n}-2)-}$ have significantly different intensities in calculated IR spectra. Bridging $\mathrm{BH}_{4}^{-}$groups inside a complex anion lead to further bands in the stretching region, situated at energies between the bands pertaining to $M-\mathrm{H}-\mathrm{B}$ bridging and $\mathrm{B}-\mathrm{H}_{t}$ stretching modes of the terminal ligands, as is the case in $\left[\mathrm{Zn}_{2}\left(\mathrm{BH}_{4}\right)_{5}\right]^{-}{ }^{-[19]}$ Complex anions can also be fingerprinted in the low-energy region, where $M-\mathrm{B}$ vibrations appear, around $300-500 \mathrm{~cm}^{-1}$, this however requires high quality spectra.

\section{Local Environment}

In a similar way, spectroscopy can fingerprint the bonding scheme itself. Bidentate bonding in a complex anion for instance is revealed by an isolated bending mode around $1400-1450 \mathrm{~cm}^{-1}$, as in $\mathrm{K}_{2} \mathrm{Mg}\left(\mathrm{BH}_{4}\right)_{4}$, for example. The orientation of $\mathrm{BH}_{4}^{-}$has significant impact on a structural description. For instance, during the structure solution of $\alpha-\mathrm{Mg}\left(\mathrm{BH}_{4}\right)_{2}$ on X-ray powder data, some $\mathrm{BH}_{4}^{-}$groups were incorrectly oriented, which resulted in the twofold axes being overlooked. The structure was initially solved in $P 6_{1}^{[14,18]}$ and later on corrected by DFT calculations to a higher symmetry, $P 6$ 22. $^{2}{ }^{23]}$ Structures maximize repulsive $\mathrm{H}$...H distances, which becomes most important in packed structures. Often there is only one possible solution (ordering scheme of $\mathrm{BH}_{4}$ ), corresponding to one specific space group, to achieve this. $H T-\mathrm{Y}\left(\mathrm{BH}_{4}\right)_{3}$, for instance, initially described in a disordered model $P m-3 m$ and solved from XPD, ${ }^{[28]}$ was later on corrected to $F m-3 c$ with NPD by ordering $\mathrm{BH}_{4}$ groups. ${ }^{[29]}$ All H...H distances are maximized in the corrected structure, resulting in an 8-fold supercell to Pm-3m. More detailed information on fingerprinting

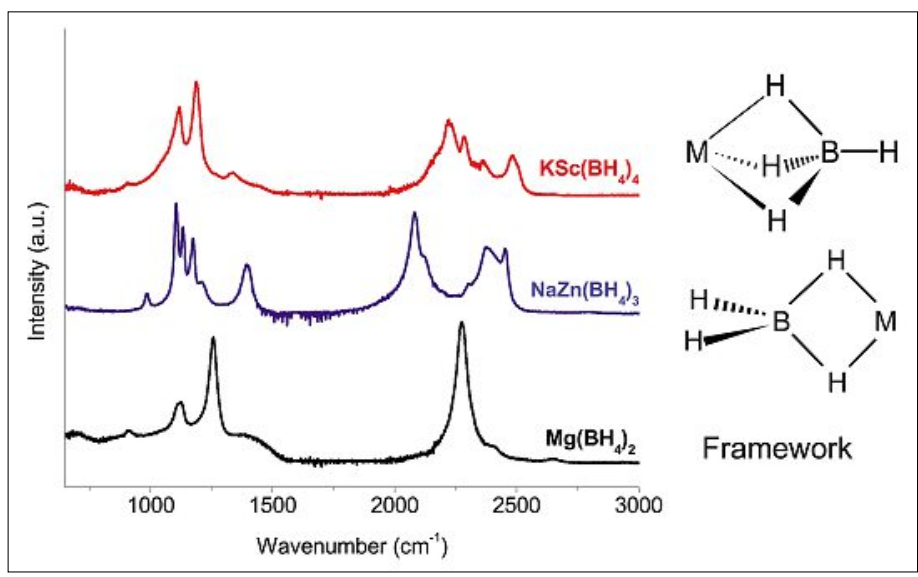

Fig. 1. Spectroscopic signatures of individual topologies and $M$... H bonding configurations. Bottom: framework $\mathrm{Mg}\left(\mathrm{BH}_{4}\right)_{2}$, middle: bidentate complex anion $\left[\mathrm{Zn}\left(\mathrm{BH}_{4}\right)_{3}\right]^{-}$, top: tridentate complex anion $\left[\mathrm{Sc}\left(\mathrm{BH}_{4}\right)_{4}\right]^{-}$. 
$M-\mathrm{H}$ bonding configurations can be found in the respective references above and has been additionally addressed very recently by D'Anna and coworkers. ${ }^{[30]}$

Solid-state NMR spectroscopy has not yet been fully exploited in light-metal hydride research, probably due to the complexity of the method, it is however commonly used by some groups, to determine how many boron positions with strongly differing local environments there are.

\section{Decomposition Mechanisms - The Role of Thermal Analysis}

From a practical point of view, the understanding of structural and chemical details should result in the accurate description of a complete storage system under working conditions. To this end, additional experimental input on a macroscopic scale is necessary, which should provide information on general system properties. In hydrogen storage systems this implies reaction enthalpies, estimation of amorphous phase contents and most importantly, decomposition mechanisms. A detailed description of the latter requires not only extensive phase analysis by means of in situ diffraction, but also the detection of all the components invisible to X-rays, such as amorphous phases and gases. Therefore, potential working systems under study should always be accompanied by thermo-analytical methods. Not only is the nature of gas release the desired number one result for any application, but simultaneously it assists powder diffraction during the process of system characterization. It is an iterative process between different methods to solve an entire system. The main contribution from TGA-MS and DTA/DSC consists in unravelling changing stoichiometries in the sample, to avoid getting mislead on unit-cell contents during reactions and thus compromise the further description. ${ }^{[31]}$ The thermodynamic character of events taking place further allows different mechanisms to be distinguished, such as displacive or order-disorder phase transitions, exothermic gas releases, endothermic melt processes, or other processes taking part in the amorphous part of the sample.

Hydrogen release kinetics can also be indirectly studied by hydrogen-deuterium gas exchange, which allows the activation energy of exchange to be determined and thus the mobility of hydrogen atoms. Deuterium and hydrogen have the same scattering power, it is therefore impossible to quantitatively relate $\mathrm{X}$-ray intensities to exchange dynamics. Precise measurements of lattice parameters can be used to track the exchange process, the respective deuteride having smaller unit cell dimensions due to the relatively heavier deuterium nucleus. ${ }^{[32]}$ This however still provides no means of quantification. In situ Raman setups are easily placed into the setup at a beamline and do provide this information, when monitoring the band intensities of Raman signals corresponding to well separated B-H and B-D vibrations. ${ }^{[33]}$

\section{Overcoming the Limits - The Unified Approach}

The characterization of all processes in a respective system requires the purposeful application of each method where it suits best. In the following we will illustrate a combined methodological approach useful for hydrogen storage systems on the basis of two recent, representative examples.

\section{System $\mathrm{KBH}_{4}-\mathrm{ZnCl}_{2}$}

This system is reported in ref. [31] and is shown here in Fig. 2. It is a classical case where various unknown phases are present in the as-synthesized sample, and X-ray powder diffraction stands no chance of decoding the $R T$ pattern on its own, containing two new compounds, as discovered later. The preparation of various samples of different ratios of starting materials is essential in these cases, as outlined above. In this case the comparison of relative signal intensities in various mixtures leads to the assignment of two sets of peaks. A quick Raman experiment reveals the stretching vibrations of $\mathrm{BH}_{4}$ to be split by approximately $300 \mathrm{~cm}^{-1}$ and points towards possible topologies based on complex anions of the kind $\left[\mathrm{Zn}\left(\mathrm{BH}_{4}\right)_{\mathrm{n}}\right]^{(\mathrm{n}-2)-}$. With this additional information the unit cell content is assigned to the indexed phases. Experience shows that, when estimating stoichiometries on borohydrides, one should usually expect atomic volumes of roughly around $40 \AA^{3}$. The $\mathrm{BH}_{4}^{-}$anion is counted as one atom. The simple fact of preparing samples from different starting compositions helps to assign stoichiometries in this 'KZn'-case, the major phase being solved as $\mathrm{KZn}\left(\mathrm{BH}_{4}\right)_{3}$ (containing $\left.\left[\mathrm{Zn}\left(\mathrm{BH}_{4}\right)_{3}\right]^{-}\right)$in $\mathrm{Zn}$-rich samples. The decomposition of $\mathrm{KZn}\left(\mathrm{BH}_{4}\right)_{3}$ at $400 \mathrm{~K}$ implicates a significant gas release, matching molar amounts of $\mathrm{B}_{2} \mathrm{H}_{6}$ and $\mathrm{H}_{2}$ (Fig. 3), which can be verified by MS. Simultaneously the second set of peaks gains in intensity, indicating thus a decomposition product. The formulation of the decomposition reaction allows the second set of peaks to be assigned to $\mathrm{K}_{2} \mathrm{Zn}\left(\mathrm{BH}_{4}\right)_{4}$. Further reactions between 400 and $445 \mathrm{~K}$ (Fig. 3) however suggest this to be an anion-substituted compound $\mathrm{K}_{2} \mathrm{Zn}\left(\mathrm{BH}_{4}\right)_{\mathrm{x}} \mathrm{Cl}_{4-\mathrm{x}}$, as the synthesis involves an incomplete metathesis reaction between $\mathrm{ZnCl}_{2}$ and $\mathrm{KBH}_{4}$. The temperaturedependent composition is refined, and the appearance of $\mathrm{KBH}_{4}$ and metallic zinc at $450 \mathrm{~K}$ (Fig. 3) suggest chlorine uptake and $\mathrm{BH}_{4}$ release in the compound, which is confirmed subsequently by quantitative analysis of the anion site during sequential Rietveld refinements. Peaks marked with a triangle at room temperature (Fig. 3 ), initially thought to belong to two different phases, can thus be related to the same solid-solution series, transforming to a higher symmetry space group as the end-member $\mathrm{K}_{2} \mathrm{ZnCl}_{4}$ is approached with temperature (Fig. 3, right). The description of this system did not require extensive input from $a b$ initio calculations, there were however some 'cosmetic' adjustments performed during the optimization of $\mathrm{KZn}\left(\mathrm{BH}_{4}\right)_{3}$, which allowed the phase to be placed into a better context to the reported $\mathrm{NaZn}\left(\mathrm{BH}_{4}\right)_{3}{ }^{[19,20]}$ These corrections concern minor but interesting details in the bidentate $\mathrm{Zn}-\mathrm{H}$ bonding configuration. Antibump-restrained Rietveld refinement results in $\mathrm{BH}_{4}$ groups with their zinccoordinating $\mathrm{H}-\mathrm{H}$ edges perpendicular to the triangular plane formed by the boron atoms in $\left[\mathrm{Zn}\left(\mathrm{BH}_{4}\right)_{3}\right]^{-}$. The Rietveld fit is good and there is no reason to doubt this geometry. DFT however tells us that these edges are all co-rotated by $16.1^{\circ}$ (Fig. 2) to minimize repulsive effects between hydrogen atoms, which then perfectly agrees with observations made on a genuine triangular $\mathrm{Al}\left(\mathrm{BH}_{4}\right)_{3}$ molecule. ${ }^{[34]}$

\section{System $\mathrm{LiBH}_{4}-\mathrm{Mg}\left(\mathrm{BH}_{4}\right)_{2}-\mathrm{ZnCl}_{2}$}

As structures become more complex, crystallographic knowledge going beyond phase characterization becomes more crucial. The recently reported compound $\mathrm{Li}_{3} M \mathrm{Zn}_{5}\left(\mathrm{BH}_{4}\right)_{15}(M=\mathrm{Mg}, \mathrm{Mn})^{[35]}$ is an instructive example where solid classical crystallography and modern $a b$ initio calculations mutually complement each other. Initially this system was chosen to synthesize theoretically studied $\mathrm{Mg}-\mathrm{Zn}$ borohydrides. ${ }^{[36]}$ It was the first attempt to work on borohydride systems containing more than two metals. Different compositional mixtures were again essential to separate the diffraction signal of the new trimetal-

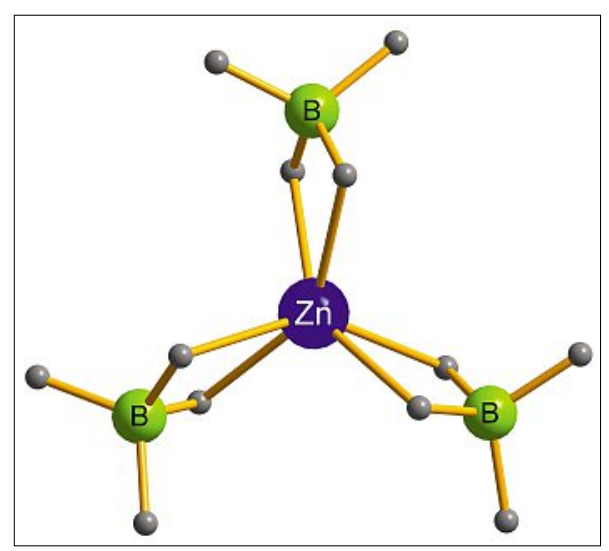

Fig. 2. Complex anion $\left[\mathrm{Zn}\left(\mathrm{BH}_{4}\right)_{3}\right]^{-}$as found in $\mathrm{KZn}\left(\mathrm{BH}_{4}\right)_{3}$, schematic view of the co-rotation of $\mathrm{BH}_{4}$-groups as optimized by DFT. 


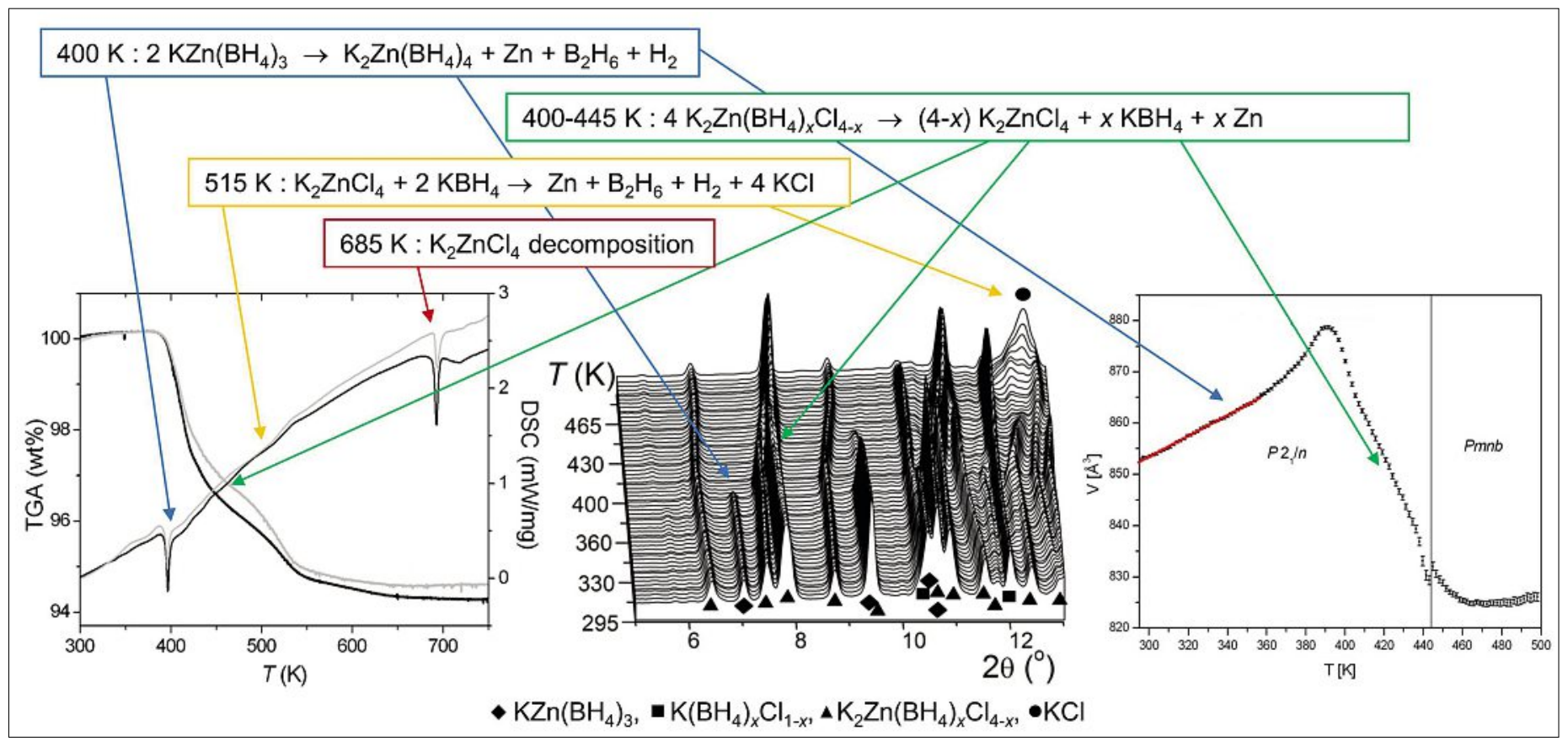

Fig. 3. Different events taking place in the system $\mathrm{KBH}_{4}-\mathrm{ZnCl}_{2}$, probed by thermal analyses TGA/DTA (left, black and grey curves corresponding to different starting ratios) and in situ $\mathrm{X}$-ray powder diffraction (middle). The temperature-dependent volume evolution of $\left.\mathrm{K}_{2} \mathrm{Zn}_{(\mathrm{BH}}\right)_{x} \mathrm{Cl}_{4-x}$ is shown on the right. Chlorine uptake starts at $390 \mathrm{~K}$ as evidenced by the decrease in volume. The chlorine end-member is reached at the symmetry change to Pmnb.

lic phase from bimetallic phases co-crystallizing in the system. In situ experiments then provided the necessary information to determine the decomposition route, which proceeds via formation of the reported bimetallic $\mathrm{LiZn}_{2}\left(\mathrm{BH}_{4}\right)_{5}$ and $\mathrm{LiBH}_{4}$, $\mathrm{Mg}\left(\mathrm{BH}_{4}\right)_{2}$. Based on this, low-resolution data then were sufficient to assign a unit cell and guess an approximate composition. During the investigation, a synthetic procedure to prepare pure $\mathrm{Mg}\left(\mathrm{BH}_{4}\right)_{2}$ was developed, ${ }^{[37]}$ which allowed us shortly after to repeat the preparations of homoleptic $\mathrm{Li}_{3} M \mathrm{Zn}_{5}\left(\mathrm{BH}_{4}\right)_{15}$, resulting in a much higher yield, and strengthening the assumed initial composition. The phase was indexed in a hexagonal cell and systematic extinctions led to a crystal structure in $P 6_{3} / \mathrm{mcm}$, the highest possible symmetry compatible with the extinction symbol $P$-c-. The decomposition mechanism and the general structural topology corroborated the proposed trimetallic character of the new phase. Occupancy-constrained refinements to ensure charge-balancing led to a mixed site $6 \mathrm{~g}$, occupied by both $\mathrm{Li}$ and $\mathrm{Mg}$. A second Li-position was loosely located in $\mathrm{BH}_{4}$ octahedral environment (Fig. 4, left) forming face-sharing channels, that suggested fast ion conduction, and its occupancy was coupled to that of $6 \mathrm{~g}$. We measured the ionic conductivity of $\mathrm{Li}_{3} \mathrm{MgZn}_{5}\left(\mathrm{BH}_{4}\right)_{15}$ with a negative result, the value not lying above a threshold of parasitic electronic conduction. This prompted us to re-inspect the occupancy of $6 \mathrm{~g}$, which now became the main problem to be solved. While neutron powder diffraction (NPD) at $15 \mathrm{~K}$ con- firmed the location of deuterium atoms as determined by restrained rigid body refinements from SP-XRD data, they provided no further information on the site mixing of $6 \mathrm{~g}$. A combined Li-Mg occupation on this site leads to a coherent scattering length of around $b_{c}=1.5 \mathrm{fm}$, as the positive $b$ of $\mathrm{Mg}$ and the negative $b$ of the natural isotope ${ }^{7} \mathrm{Li}$ partially cancel out in this mixture. The scattering lengths of ${ }^{11} \mathrm{~B}$ and $\mathrm{D}$ amount to 6.65 and $6.671 \mathrm{fm}$, respectively, thus becoming the main scatterers in NPD. Sequential Rietveld refinements of in situ data are a useful tool and commonly employed in complex hydrides to detect slight changes of lattice parameters, peak shapes or phase compositions, indicative of compositional changes or substitutions of the involved phases. The energy of mixing on a given crystallographic site is an entropic contribution. Therefore, a chemically disordered site tends to change composition as a function of temperature. We inspected diffraction data at intervals of $1.5 \mathrm{~K}$ but the stoichiometry of $\mathrm{Li}_{3} M \mathrm{Zn}_{5}\left(\mathrm{BH}_{4}\right)_{15}$ does not vary at all between $150 \mathrm{~K}$ and the decomposition of the compound at $393 \mathrm{~K}$, nor does the chemical composition of $6 \mathrm{~g}$. This suggested that only an average view of the chemically disordered Li-Mg occupancy on $6 \mathrm{~g}$ is seen by diffraction. This has crystallographic consequences. Ordering of $6 \mathrm{~g}$ breaks the hexagonal symmetry down to orthorhombic. Fully ordered structural models can be studied by periodic solidstate $a b$ initio calculations and can provide valuable information when a choice of space group needs to be reconsidered towards the end of a structural characterization. This said, starting models are generated and biased by diffraction. In the case of $\mathrm{Li}_{3} \mathrm{MgZn}_{5}\left(\mathrm{BH}_{4}\right)_{15}$, calculations on ordered orthorhombic models show that the minimal optimized energies (per unit cell) correspond to three distributions that maximize $\mathrm{Mg}-\mathrm{Mg}$ distances. Superposing these distributions with equal probability results in the symmetry $P 6_{3} / \mathrm{mcm}$ and in $6 \mathrm{~g}$ $\mathrm{Mg}-\mathrm{Li}$ occupancies as determined and refined with diffraction data. From a crystalchemical point of view, these findings justify an ordered description of the structure. However, they do not yet provide an explanation for the non-conducting character of the phase. Another very useful correction that DFT finds in this case concerns the second (octahedral) Li position, 2b. During optimizations, lithium atoms start moving from the center of the octahedron to the octahedral faces shared by neighboring polyhedra along the $c$-direction. Positioning Li in this triangular environment (Fig. 4) and recalculating results in significantly lower energies. DFT has thus corrected a structural feature invisible to diffraction (both SR-XPD and NPD place Li in the octahedron). The topological description changes slightly, rendering the general picture more coherent. Lithium is commonly found in a tetrahedral environment in borohydrides. The triangular coordination is however better adapted to its small size than the octahedral one. The stronger bonding interactions in $\mathrm{Li}\left(\mathrm{BH}_{4}\right)_{3}{ }^{2-}$ fragments are compatible with the zero conductivity. 


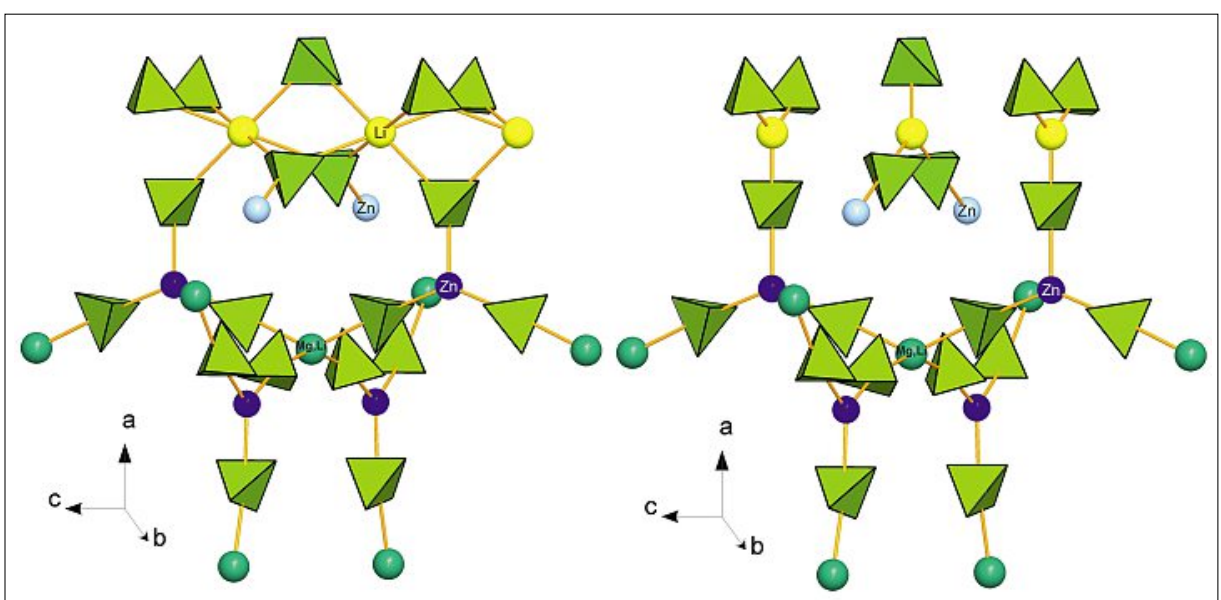

Fig. 4. Locations of lithium on site $2 b$ as determined by X-ray and neutron powder diffraction (left) and as optimized by density function theory calculations (right).

\section{Some Words on DFT}

While $a b$ initio calculations are still commonly regarded as inadequate in predicting complex hydride crystal structures, their value in providing details and thus complementing the experimental information is becoming more obvious. But DFT, when applied correctly and exhaustively to the problem, goes beyond the validation of experimental structures. Density functional theory is not only capable of orienting complex groups properly and providing accurate inter-atomic distances and angles, it can in some cases correct features going beyond the local structure.[22] However, there are a number of relevant pitfalls when applying DFT to borohydrides. Additionally one must keep in mind that if used to validate powder structures, one is employing a far more precise method to 'validate' a less precise one. Both may be inaccurate though. Thus, optimizing a structure may not necessarily equate to making it more accurate. Well-founded crystallographic and chemical analysis will always remain indispensable. Weak interactions need to be accounted for correctly in $a b$ initio calculations and dispersion corrections are still in development, as are the codes for the solid state itself. $\mathrm{Mg}\left(\mathrm{BH}_{4}\right)_{2}$ has received most of the theoretical attention, and, amongst innumerable attempts, has been predicted (post experiment) only once correctly upon the inclusion of van der Waals corrections. ${ }^{[38]}$ Complex hydrides are often either chemically disordered, or show some extent of positional site disorder on both cation and anion sites. Most importantly, $\mathrm{BH}_{4}$ is known to be inherently dynamic, subject to different jump motions also in ordered phases. Any kind of disorder presents an obstacle for DFT. However, chemical order may be modeled, e.g. as shown above, by different subgroups, and rotational disorder may be attempted to model in supercells. ${ }^{[39]} \mathrm{A}$ more sophisticated tool may be to implement molecular dynamics in periodic DFT calculations. ${ }^{[40]} \mathrm{LiBH}_{4}$ is a reference testcase for the theoretical 'hydrides world'. It undergoes an orthorhombic-hexagonal transition, Pnma $-P_{3} m c$ at $387 \mathrm{~K}$ that involves polar disorder of $\mathrm{BH}_{4}^{-}$. Years of effort have not succeeded in modeling the $H T$ phase. Very recently this was achieved by including dynamics in a combined MD-DFT approach. ${ }^{[41]}$ Experimental tools more adapted to characterize properties coupled to dynamic disorder are solid-state NMR spectroscopy ${ }^{[42,43]}$ and quasi-elastic neutron scattering. ${ }^{[44,45]}$ Both are being applied to borohydrides, and the former has recently also been coupled to $a b$ initio calculations. ${ }^{[46]}$ These methods can also be considered as extremely sophisticated options to decide on structural polarity, if it depends on the orientation of $\mathrm{BH}_{4}^{-}$groups, inaccessible to $\mathrm{X}$-ray diffraction. One inconvenient side-effect of the disordered nature of complex hydrides is that even experimentally measured room-temperature structures rarely or never represent the ground state itself. DFT calculations are commonly performed at $0 \mathrm{~K}$ where dynamics largely freeze. This obviously severely biases structural predictions. For structural corrections/optimizations, the choice needs to be taken of either fixing the unit cell to experimentally obtained lattice constants (preferably on powder samples) or optimizing the cell geometry along with atomic positions. It is up to the user and his/her experience to decide which is more efficient, depending on the specific problem to be addressed. Our own experience tells us that DFT can be of tremendous use, in spite of all its constraints, especially when working with hydrides that have significant differences between scattering powers of contained atoms. For instance, while $\mathrm{LiBH}_{4}$ could be fully refined anisotropically with unconstrained hydrogen atom positions, ${ }^{[8]}$ the structures of a recent system, $\mathrm{CsBH}_{4}-\mathrm{LiBH}_{4}{ }^{[24]}$ needed to be solved on heavy atoms only, subsequently introducing the lighter components. This corresponds to the 'heavy-atom' method or Patterson methods in reciprocal space. In these cases DFT can be valuable to make a correct subgroup choice, if the structure has been solved in a supercell. We introduced spectroscopy as a fingerprinting tool for the metal-hydrogen coordination scheme. This is a fact that is readily validated with DFT, as theoretical IR spectra are easily extracted from an optimization, provided the structure is stable (no imaginary frequencies). Even more so this is the case for inelastic neutron scattering spectra, ${ }^{[47]}$ which are however tedious to analyze on powders, that do not allow the measurement of specific phonon branches.

\section{Outlook}

It is certain that in the future potentially useful systems in all energy storage fields, be it hydrogen, batteries, or other electrochemical approaches, will involve highly complex and dynamic systems, which are subject to metastability and changing thermodynamic equilibria, particle sizes and crystallinity. The setups at synchrotron beamlines are evolving quickly and it will become increasingly feasible to study systems under working conditions. Structure solution and complete structural characterization will always be at the upmost front to characterizing these systems. Powder diffraction is and will surely remain the most amenable method due to the broad spectrum of information obtainable and the simplicity of in situ experiments coupled to complementary methods such as EXAFS, IXS, vibrational spectroscopy or TGA/ DTA-MS just to name the most useful ones for this field. Electron diffraction has developed rapidly in recent time to challenge powder diffraction, and has already been successfully applied to borohydrides. ${ }^{[48]}$ However, its inherent local sampling on a nanometer scale and substantially complex experimental setup inhibiting in situ coupling to other methods pose serious problems for hydrogen storage-oriented experiments. Additionally, the sensitivity of soft matter to the electron beam will limit the method to special applications such as symmetry determination. From a crystallographic point of view, ingenious developments such as direct space methods or charge-flipping are complemented by first-principles assisted structure solution that has proven to work well for relevant systems $^{[49]}$ and represents one more step in unifying theoreticians and experimentalists. Especially the prediction of new systems will be a common ground for both research fields. Not only can experimentalists 
provide theoreticians with information for calculations, but predictions on whole systems can also be confirmed by experiment as was recently illustrated on potassium silanides, ${ }^{[50]}$ and which is the original idea of physical science in general.

Received: January 4, 2014

[1] Walker G., Ed., 'Solid-state hydrogen storage, materials and chemistry', Woodhead Publishing Limited, Cambridge, 2008.

[2] http://www.fuelcelltoday.com, Analyst view: Fuel Cells and Submarines, 2013. (see also http://en.wikipedia.org/wiki/Type_212_ submarine)

[3] 'Targets for Onboard Hydrogen Storage Systems for Light-Duty Vehicles', US Department of Energy Office of Energy Efficiency, Renewable Energy and The FreedomCAR and Fuel Partnership, 2009.

[4] http://uscar.org/guest/teams/19/U-S-DRIVEHydrogen-Storage-Tech-Team.

[5] H. Hagemann, R. Černý, Dalton Trans. 2010, $39,6006$.

[6] J. Huot, D. B. Ravnsbæk, J. Zhang, F. Cuevas, M. Latroche, T. R. Jensen, Prog. Mater. Sci. 2013, 58, 30 .

[7] Y. Filinchuk, R. Černý, H. Hagemann, Chem. Mater. 2009, 21, 925.

[8] Y. Filinchuk, D. Chernyshov, R. Čeerný, J. Phys. Chem. C 2008, 112, 10579.

[9] L. H. Rude, M. Corno, P. Ugliengo, M. Baricco, Y.-S. Lee, Y. W. Cho, F. Besenbacher, J. Overgaard, T. R. Jensen, J. Phys. Chem C 2012, 116, 20239.

[10] Y. E. Filinchuk, K. Yvon, G. P. Meisner, F. E. Pinkerton, M. P. Balogh, Inorg. Chem. 2006, 45, 1433.

[11] T. R. Jensen, T. K. Nielsen, Y. Filinchuk, J. E. Jørgensen, Y. Cerenius, E. MacA. Gray, C. Webb, J. Appl. Cryst. 2010, 43, 1456.

[12] T. Friščič, I Halasz, P. J. Beldon, A. M. Belenguer, F. Adams, S. A. J. Kimber, V. Honkimäki, R. E. Dinnebier, Nature Chem. 2012, 5, 66.

[13] Y. Filinchuk, A. Talyzin, D. Chernyshov, V. Dmitriev, Phys. Rev. B 2007, 76, 092104.
[14] J.-H. Her, P. W. Stephens, Y. Gao, G. L. Soloveichik, J. Rijssenbeck, M. Andrus, J.-C. Zhao, Acta Cryst. B 2007, 63, 561.

[15] L. B. McCusker, C. Baerlocher, Chima, 2014, $68,19$.

[16] R. Černý, Z. Kristallogr. 2008, 223, 607.

[17] V. Favre-Nicolin, R. Černý, J. Appl. Cryst. 2002, 35, 734. Also, http://objcryst.sourceforge. net/Fox

[18] R. Černý, Y. Filinchuk, H. Hagemann, K. Yvon, Angew. Chem. Int. Ed. 2007, 46, 5765.

[19] R. Černý, K. C. Kim, N. Penin, V. D’Anna, H. Hagemann, D. S. Scholl, J. Phys. Chem. C 2010, 114, 19127.

[20] D. Ravnsbæk, Y. Filinchuk, Y. Cerenius, H. J. Jakobsen, F. Besenbacher, J. Skibsted, C. M. Jensen, T. R. Jensen, Angew, Chem., Int. Ed. 2009, 48,6659 .

[21] R. Cerný, Y. Filinchuk, Z. Kristallogr. 2011, 226,882 .

[22] I. Lindemann, R. D. Ferrer, L. Dunsch, Y. Filinchuk, R. Cerný, H. Hagemann, V. D’Anna, L. M. Lawson, Chem. Eur. J. 2010, 16, 8707.

[23] B. Dai, D. S. Scholl, J. K. J. Johnson, J. Phys. Chem. 2008, 112, 4391.

[24] P. Schouwink, L. Smrčok, R. Černý, in preparation.

[25] I. Dovgaliuk, V. Ban, Y. Sadikin, R. Černý, L. Aranda, M. Devillers, Y. Filinchuk, J. Phys. Chem. C 2014, 118, 145.

[26] D. Reed, D. Book, Curr. Opin. Solid. St. M. 2011, 15, 62

[27] E. Callini, A. Borgschulte, A. J. RamirezCuesta, A. Züttel, Dalton Trans. 2013, 42, 719

[28] D. Ravnsbæk, Y. Filinchuk, R. Černý, M. B. Ley, D. Haase, H. J. Jakobsen, J. Skibsted, T. R. Jensen, Inorg. Chem. 2010, 49, 3801.

[29] C, Frommen, N. Aliouane, S. Deledda, J. E. Fronneløp, H. Grove, K. Lieutenant, I. LlamasJansa, S. Sartori, M. H. Sørby, B. Hauback, J. Alloys Comp. 2010, 496, 710.

[30] V. D’Anna, L. M. Lawson Daku, H. Hagemann, J. Alloys. Comp. 2013, 580, S122.

[31] R. Černý, D. Ravnsbæk, P. Schouwink, Y. Filinchuk, N. Penin, J. Teyssier, L. Smrčok, T. R. Jensen, J. Phys Chem C 2012, 116, 1563.

[32] H. Dammak, E. Antoshchenkova, M. Hayoun, F. Finocchi, J. Phys.: Condens. Matter 2012, $24,435402$.
[33] A. Borgschulte, A. Züttel, P. Hug, A.-M. Racu, J. Schoenes, J. Phys. Chem. A 2008, 112, 4749.

[34] A. Al-Kahtani, D. L. Williams, J. W. Nibler, S. W. Sharpe, J. Phys. Chem. A 1998, 102, 537.

[35] R. Černý, P. Schouwink, Y. Sadikin, K. Stare, L. Smrčok, B. Richter, T. R. Jensen, Inorg. Chem. 2013, 52, 9941.

[36] E. Albanese, G. Kalantzopoulos, J. G. Vitillo, E. Pinatel, B. Civalleri, S. Deledda, S. Bordiga, B. Hauback, M. Baricco, Book of Abstracts, MH212, Kyoto 2012, 179.

[37] Y. Filinchuk, B. Richter, T. R. Jensen, V. Dmitriev, D. Chernyshov, H. Hagemann, Angew. Chem. Int. Ed. 2011, 50, 11162.

[38] A. Bil, B. Kolb, R. Atkinson, D. G. Pettifor, T. Thonhauser, A. N. Kolmogorov, Phys. Rev. B 2011, 83, 224103.

[39] L. Smrčok, in 'Uniting Electron Crystallography and Powder Diffraction', Editors U. Kolb, K. Shankland, L. Meshi, A. Avilov, W. David, Springer, Heidelberg, 2011, p. 165.

[40] R. Car, M. Parrinello, Phys. Rev. Lett. 1985, 55, 2471.

[41] P. C. Aeberhard, K. Refson, W. I. F. David, Phys. Chem. Chem. Phys. 2013, 15, 8081.

[42] O. A. Babanova, A. V. Soloninin, A. V. Skripov, D. B. Ravnsbæk, T. R. Jensen, Y. Filinchuk, J. Phys. Chem. C 2011, 115, 10305.

[43] N. Verdal, T. J Udovic, J. J. Rush, H. Wu, A. V. Skripov, J. Phys. Chem. C 2013, 117, 12010.

[44] P. Martelli, A. Remhof, A. Borgschulte, R. Ackermann, T Straessle, J. P. Embs, M. Ernst, M. Matsuo, S.-I. Orimo, A. Zuettel, J. Phys. Chem. A 2011, 115, 5329.

[45] A. Remhof, Z. Lodziana, P- Martelli, O. Friedrichs, A. Zuettel, A. V. Skripov, J. P. Embs, T. Straessle, Phys. Rev. B 2010, 81, 214304.

[46] F. Franco, M. Baricco, M. R. Chierotti, R. Gobetto, C. Nervi, J. Phys. Chem. C 2013, 117, 9991.

[47] S.F. Parker, Coordin. Chem. Rev. 2010, 254, 215.

[48] J. Haderman, A. Abakumov, S. Van Rompaey, T. Perkisas, Y. Filinchuk, G. Van Tendeloo, Chem. Mater. 2012, 24, 3401.

[49] B. Meredig, C. Wolverton, Nature Mater. 2013, $12,123$.

[50] J.-N. Chotard, W. S. Tang, P. Raybaud, R. Janot, Chem. Eur. J. 2011, 17, 12302. 